\title{
Dynamic Investigation of Metal-support Interactions in Heterodimer Nanoparticles by in situ Transmission Electron Microscopy
}

\author{
Volkan Ortalan ${ }^{1}$, Chang Wan Han ${ }^{1}$, Jefferey Greeley ${ }^{2}$, Chao Wang ${ }^{3}$, Fabio H Ribeiro ${ }^{2}$, Cory Milligan ${ }^{2}$, \\ Tej Choksi ${ }^{2}$, Paulami Majumdar ${ }^{2}$ and Michael Manto ${ }^{3}$ \\ 1. School of Materials Engineering, Purdue University, West Lafayette, IN, USA \\ 2. School of Chemical Engineering, Purdue University, West Lafayette, IN, USA \\ 3. Chemical and Biomolecular Engineering, Johns Hopkins University, Baltimore, MD, USA
}

Catalysts are critical components of many current technologies and essential components of sustainable energy systems ranging from fuel cells and batteries to turning biomass into useful chemicals or fuels by enabling reactions to be guided quickly and efficiently along desirable pathways rather than those that are inefficient or lead to unwanted byproducts. Fundamental investigations of catalyst structures and the mechanisms of catalytic reactions requires characterization imaging of surfaces at the atomic scale and probing the structures and energetics of the reacting molecules as they function under reaction condition on varying time and length scales. High-angle annular dark field (HAADF) STEM is an indispensable technique for analyzing heterogeneous catalysts, in particular those comprising high atomic number $(Z)$ metallic nanoparticles (NPs) dispersed on low-Z supports ${ }^{1}$. Readily interpretable atomic-scale Z-contrast imaging with high spatial resolution spectroscopy, including X-ray energy dispersive spectroscopy (EDS) and electron energy loss spectroscopy (EELS), allows researchers to investigate structural information such as dimensions, morphologies, and size distribution of catalytic NPs as well as their material chemistry (e.g., chemical composition, bonding of catalytic particles with supports at interface, etc.).

The strength of metal-support bonding in heterogeneous catalysts determines their thermal stability, therefore, a tremendous amount of effort has been expended to understand metal-support interactions ${ }^{2-4}$. Herein, we report the discovery of an anomalous "strong metal-support bonding" between gold nanoparticles and "nano-engineered" $\mathrm{Fe}_{3} \mathrm{O}_{4}$ substrates by in-situ microscopy (Figure 1). The in situ heating experiments performed (up to $500{ }^{\circ} \mathrm{C}$ ) under reducing conditions using aberration-corrected TEM and Protochips Co. Aduro ${ }^{\mathrm{TM}}$ MEMS-based heating technology resulted in very interesting wetting behavior (Figure 2). In some of the heterodimer structures, we also observed epitaxial wetting behavior suggesting that there are special crystal facets allowing epitaxial wetting between $\mathrm{Au}$ and $\mathrm{Fe}_{3} \mathrm{O}_{4}$. This epitaxial wetting mechanism also relatively slower than the one with no epitaxial relationship between the oxide and support. During in-situ vacuum annealing of $\mathrm{Au}-\mathrm{Fe}_{3} \mathrm{O}_{4}$ dumbbell-like nanoparticles, synthesized by the epitaxial growth of nano- $\mathrm{Fe}_{3} \mathrm{O}_{4}$ on $\mathrm{Au}$ nanoparticles, the gold nanoparticles transform into monolayered gold films and wet the surface of nano- $\mathrm{Fe}_{3} \mathrm{O}_{4}$, as the surface reduction of nano- $\mathrm{Fe}_{3} \mathrm{O}_{4}$ proceeds. Moreover, DFT calculations show progressively stronger binding of $\mathrm{Au}$ films with the reduction of the iron oxide support. This phenomenon results from a unique coupling of the size-and shape-dependent high surface reducibility of nano- $\mathrm{Fe}_{3} \mathrm{O}_{4}$ and the extremely strong adhesion between $\mathrm{Au}$ and the reduced $\mathrm{Fe}_{3} \mathrm{O}_{4}$.

In summary, we have employed a combination of in-situ TEM, XPS, and DFT calculations to obtain a mechanistic understanding of the wetting behavior of the gold nanoparticles in dumbbell-like nanoparticle assemblies with completely gold covered iron oxide domains during high temperature annealing. This drastic morphological change of $\mathrm{Au}$ is a direct evidence of the strong metal-support bonding (SMSB) between gold and iron oxide, which occurred during the vacuum annealing. In this 
presentation, we will discuss the origin of the SMSB in the $\mathrm{Au}-\mathrm{Fe}_{3} \mathrm{O}_{4}$ heterodimer nanoparticles which has been obtained utilizing a combination of state-of-the-art experimental (in-situ TEM, EELS, tomography and XPS) and computational modeling techniques. Moreover, we will expand the discussion with other metal-support heterodimer nanoparticle systems that we studied recently to emphasizes the significance of the size and morphology control of the metal oxide support for tuning the metal-support bonding [5].

References:

[1] P.D. Nellist PD et al, Science 305, (2004), p.1741.

[2] Farmer, J. A. \& Campbell, C. T. Science 80, (2010). 329, 933.

[3] Tauster, S. J. et al, Science 211, no 4487, (1981), p.1121.

[4] Datye, A. K., et al, Journal of Catalysis 155, no. 1 (1995), p.148.

[5] This work was supported by the Designing Materials to Revolutionize and Engineer our Future (DMREF) program of the National Science Foundation (CBET-1437219).

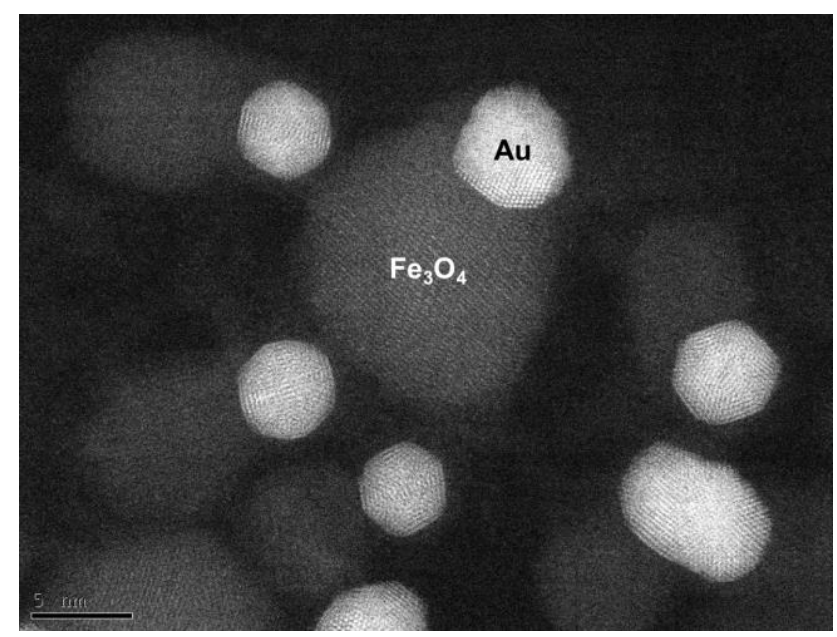

Figure 1. HAADF-STEM images of $\mathrm{Au}-\mathrm{Fe}_{3} \mathrm{O}_{4}$ heterodimer nanoparticle structures.

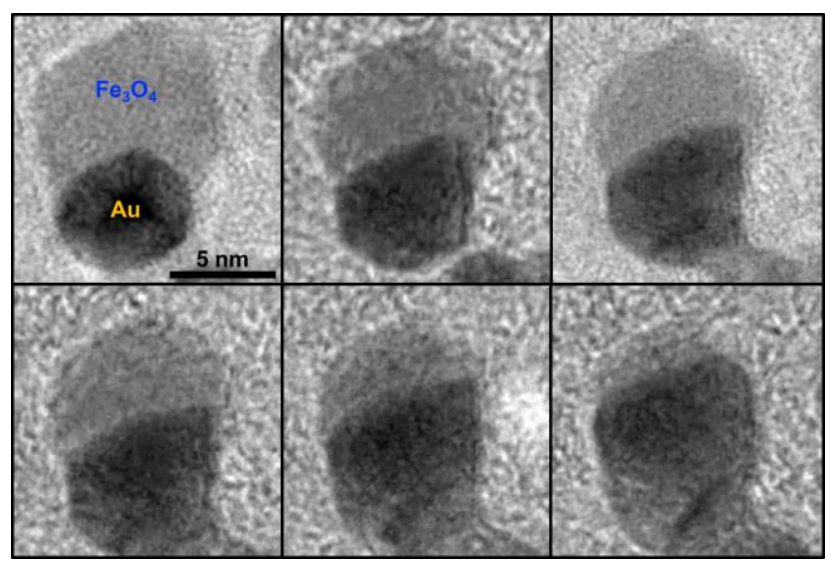

Figure 2. Consecutive in situ heating TEM images showing the epitaxial wetting of Au on nano- $-\mathrm{Fe}_{3} \mathrm{O}_{4}$ 